\title{
FLSmidth Colossal filter - demonstration plant
}

\author{
J Chaponnel FLSmidth USA Inc., USA
}

T Wisdom FLSmidth USA Inc., USA

\begin{abstract}
FLSmidth has installed and is operating a tailings dewatering demonstration plant at a concentrator in the Atacama region in Chile. The intention of the plant is to demonstrate the full-scale capabilities of our newest large-pressure filter for tailings, dubbed the Colossal filter press. It is intended to demonstrate the capabilities of one large filter press, where a number of the smaller filters would be required to process the full plant tailings. The dewatering plant is installed along the tailings line after the tailings thickeners in the vicinity of the tailings pond.

The plant is designed to dewater 10,000 tpd of filtered tailings. The filtered tailings contain roughly 82 wt $\%$ solids, compared with approximately $52 \mathrm{wt} \%$ solids in the thickened tailings feed stream. Clarified filtrate is pumped to a clean water pond for reuse in the concentrator. Components of the plant include a feed slurry tank, filter feed pumps, one Colossal filter with drip trays, an innovative hybrid apron belt feeder, bridge crane, takeaway conveyor, radial stacker, filtrate equalisation tank, clarifier, floc system, pumps, and instrumentation.

With the Colossal filter installed, commissioned and in operation, FLSmidth provides a paper containing the results that have been gathered thus far, with the intention of presenting all of the results that have been gathered prior to the 21st International Seminar on Paste and Thickened Tailings. The design philosophies, commissioning challenges and operational data associated with building and operating a large format filter are reviewed.
\end{abstract}

Keywords: tailings, dewatering, filtration

\section{Introduction}

In the years since the last catastrophic tailings dam failure, the mining industry has seen immense changes to the regulations, procedures and guidelines under which it operates. While each region has its own regulatory bodies and governance, there are common themes within the rules that each mining region sets forth. The main message that is being sent to the industry globally, however, is that mine operators need to dispose of their tailings responsibly, and in a way that mitigates the risk of further tailings dam failures in the years to come.

In addition to tailings disposal regulations, mining operators around the world are also seeing restrictions implemented which limit the amount of fresh water that can be used for mining operations. In arid regions, this is becoming a significant issue as mining companies are being forced to look for other sources of water, some opting to desalinate ocean water, before pumping it hundreds of kilometres in distance and thousands of metres in elevation to the mine site. With the cost of clean water increasing, the profitability of the mines is suffering as a result.

In an attempt to mitigate the risk of tailings dam failures and to reduce operating costs, many mining companies are now completing pre-feasibility and feasibility studies to compare the costs of filtered tailings to traditional methods of tailings disposal, with the increased cost of water and stringent safety regulations.

While there are a growing number of filtered tailings installations around the world, the majority of these are small operations with low-throughput tonnages. With a large number of concentrator plants being slated to process over 100,000 tpd of ore, FLSmidth identified the need for a large-scale filter press to 
reduce the number of operating filters that would be needed within the tailings filtration building. To reduce the total capital and operating expenses traditionally associated with filtered tailings, the FLSmidth Colossal filter was developed. To achieve this reduction in cost, the filter was designed to process twice the mass of tailings per hour per filter of its predecessors which were available in the market at that time, meaning that half the number of filters would need to operate and be maintained for any given throughput tonnage.

As with any new technology in the mining industry, the Colossal filter would need to be tested and operated before it would be accepted and trusted within the industry. To prove the filter's capability, FLSmidth partnered with a large mining company located in the Atacama region of Chile to construct a demonstration plant that would dewater the tailings from the nearby concentrator plant.

The demonstration plant was designed to meet the following criteria as set out by the client:

- 10,000 tpd of throughput.

- Cake solids content of $82 \mathrm{wt} \%$ to meet geotechnical requirements.

- Filter availability of greater than $85 \%$.

With the demonstration plant now commissioned and operating data being generated, the intent of this paper is to provide details surrounding the operation of the demonstration plant including the successes, the hurdles that have been faced, and the lessons learned during this time.

\section{Demonstration plant operating philosophy}

The system is fed by the existing thickened tailings stream from the tailings thickener underflow. A bleed stream from the main tailings slurry flow is fed to the feed tank with a target a solids content of $52 \mathrm{wt} \%$. Two filter feed pumps operate in series at a feed pressure of up to 15 bar to fill the filter and form fully consolidated cakes. The feed pump variable frequency drives (VFDs) are controlled by the automation system that controls the entire plant.

Once the filtration step is complete, the filter control system automatically configures the process valves to perform a core wash and core blow to remove unconsolidated cake from the feed eyes of the filter plates. This material exits the filter to the core separation tank, before draining to the floor sump.

The process valves are then configured to perform a 'grid sweep' of the filter plates to remove any residual water that remains in the pip surface of the filter plates prior to opening the filter. This process is called fast filtering and produces a saturated filter cake formed at 15 bar terminal pressure.

The drip trays are then opened and the clamping cylinders retracted within the follower. The crosshead then retreats, opening the plate stack completely like an accordion in less than one minute. The volume of filter cake discharged during each cycle is approximately $40 \mathrm{~m}^{3}$ of material at $82 \mathrm{wt} \%$ solids.

The discharged material falls to the hybrid apron belt (HAB) feeder that is located below the filter. The HAB feeder is designed to accomplish two things. Firstly, it is designed to handle the impact of the $40 \mathrm{~m}^{3}$ of tailings falling upon it in under one minute, and secondly it is designed to meter the $40 \mathrm{~m}^{3}$ of material to the takeaway conveyor in a uniform fashion. The control system controls the $H A B$ at a speed which matches the cycle time of the filter, ensuring that the flow of material from the $H A B$ to the takeaway conveyor is seamless throughout continuous operation. The HAB is designed with vertical chute walls to eliminate potential bridging of filter cakes.

The filter then completes a cloth shaking sequence to remove any debris from the filter media. The drip trays are then closed, and the flood wash system is activated to wash all filter cloths simultaneously for a period of approximately one minute. Once the flood wash step is complete the filter will automatically begin its next filtration cycle. 
As the filter requires a source of clean water for cloth washing, a small inclined plate clarifier was installed to clean the filtrate and wash water from the filter for reuse in washing activities. A small flocculent make-down and injection plant has been installed to produce and provide the clarifier with a source of flocculent.

\section{Plant construction and operation}

The tailings filtration demonstration plant was designed by FLSmidth in conjunction with a third party engineering company located in Chile. Construction of the demonstration plant and installation of the equipment (Figure 1) was managed by the mining company. The commissioning of the plant was completed by FLSmidth operators, who will continue to operate and maintain the facility.

While the plant today is a successful implementation of large-scale filtered tailings, there were many hurdles faced and lessons learned throughout the construction and commissioning period. The following highlights some of the challenges that were faced, the items that would be done differently, and technical challenges that were tested and proven successful during the start-up of the Colossal filter demonstration plant.

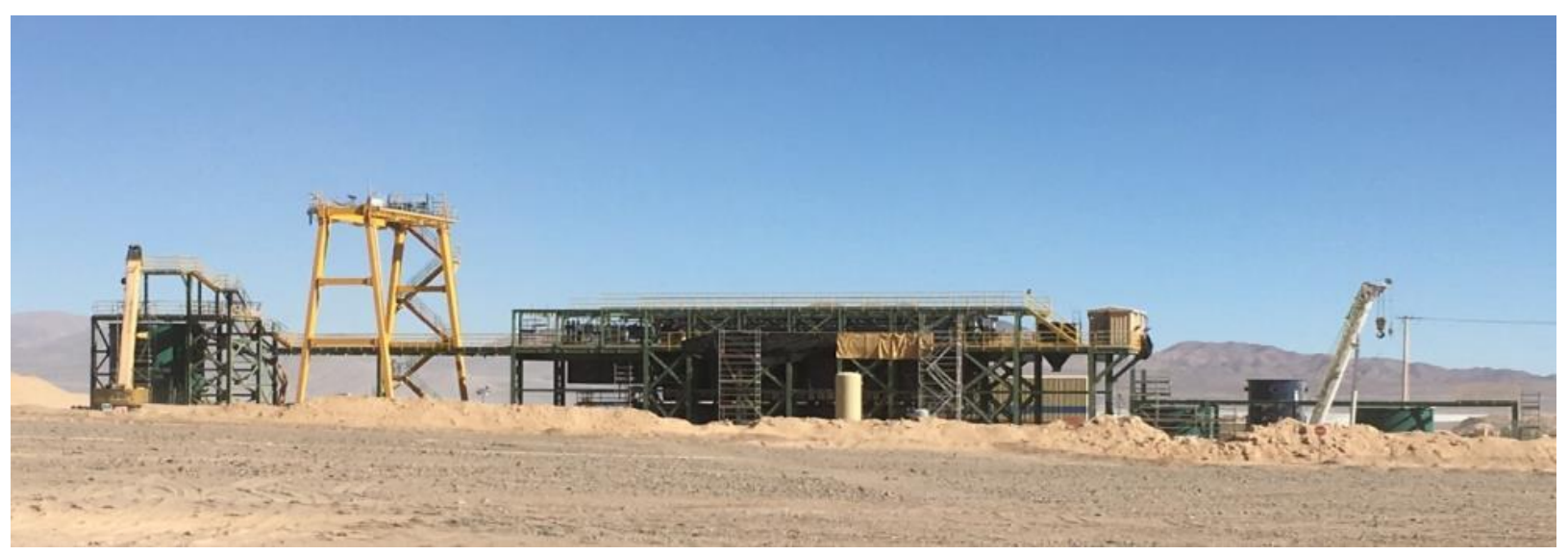

Figure 1 The Colossal filter press demonstration plant

\subsection{Engineering of the Colossal filter}

When designing such a large filter, there were several engineering challenges that needed to be overcome. The filter needed to be able to dewater $\sim 10,000 \mathrm{tpd}$ of tailings material while maintaining a filter availability of $>85 \%$. This level of advancement in technology and capacity comes with certain problems and risks to be resolved for long-term reliable operation. Some of the risks that we identified were as follows:

- High-speed mechanical systems are required to further reduce quantity of filters. Massive components needed to support pressure filtration of this magnitude must be actuated at relatively high speed. Total mechanical functions and cloth washing should not exceed a total of three minutes.

- Large and heavy plates require high-strength links to open the filter. Link failure can cause catastrophic damage to plate systems during opening of the filter plate stack.

- Distribution of feed slurry into the chamber to provide a homogenously formed filter cake (cake densities and thickness are uniform over the entire area) preventing plate failure in part by minimising potential for pressure differences from one chamber to the next.

- Large flows and high filtration rates require very large filter feed pumps, tending to push motor voltages into the medium voltage range. Since variable frequency drives are used to control pumping profile for best cake formation attributes, medium voltage can become a significant capital expenditure impact. 
Each of these issues was reviewed, leading to numerous design advancements. The resulting filter has the following characteristics as seen in Table 1. The prototype of the new design is shown in Figure 2.

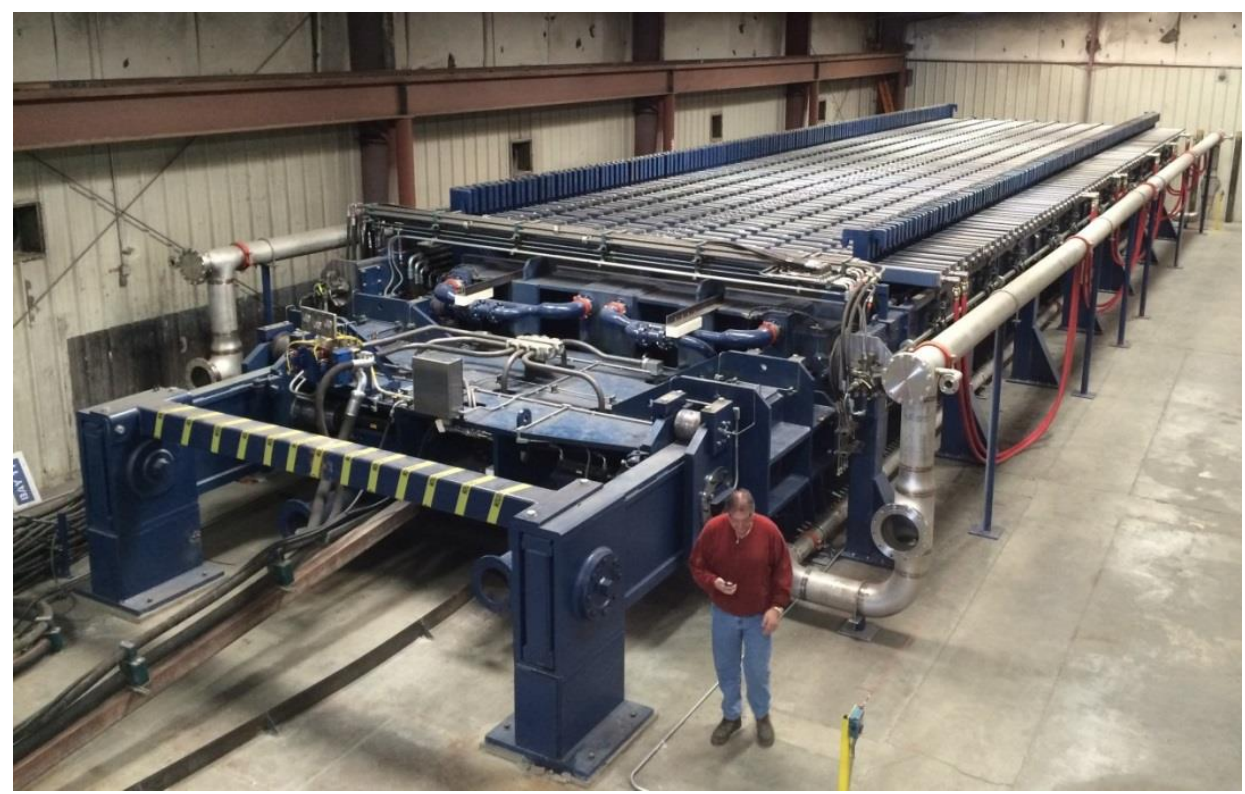

Figure 2 The Colossal filter press at the fabrication facility

Table 1 Colossal filter specifications

\begin{tabular}{ll}
\hline Design envelope specifications & \\
\hline Plate dimension $(\mathrm{mm})$ & $2,000 \times 4,000$ \\
Max. quantity of chambers & 152 \\
Max. filtration area per filter $\left(\mathrm{m}^{2}\right)$ & $>2,000$ \\
Filter volume $\left(\mathrm{m}^{3}\right)$ & $>40$ \\
Max. filtration pressure (bar) & 15 \\
\hline
\end{tabular}

\subsubsection{High-speed mechanical systems}

The Colossal filter was designed to have a total mechanical time of no longer than three minutes. This meant that the filter had to be able to complete a core blow, eye flush, open the filter, shake the filter cloths, wash the filter cloths, and close, all within this time frame. To achieve this, the filter needed to be able to open the 152 plates in less than 60 seconds.

The speed in which the heavy mechanical components operate means that high levels of stress are placed on the major weldments that make up the filter. To ensure that the filter was designed for infinite life, finite element analysis (FEA) was completed. Once the filter was assembled, strain gauges were fitted to salient areas on the weldments and the filter was operated. The strain gauge data was then compared to the FEA results, which confirmed that the filter was designed for the loads and stresses that it would be subjected to.

\subsubsection{Carbon fibre plate links}

When designing a filter that opens all filter plates consecutively in less than one minute, a tremendous amount of force is imparted upon the links that are used to connect one plate to the next. This force increases as each consecutive plate begins to shift. To withstand these forces, the standard metallic or chain type links used in smaller format filters would not be suitable for this application. 
An R\&D project was started to develop a carbon fibre link system (Figure 3) that would offer cost-effective and reliable performance for this application. Several designs were built and destructively tested, to ensure the links would be able to withstand the tension in which they would be placed. After three design changes, the links that are fitted to the Colossal filter were tested and proven successful.

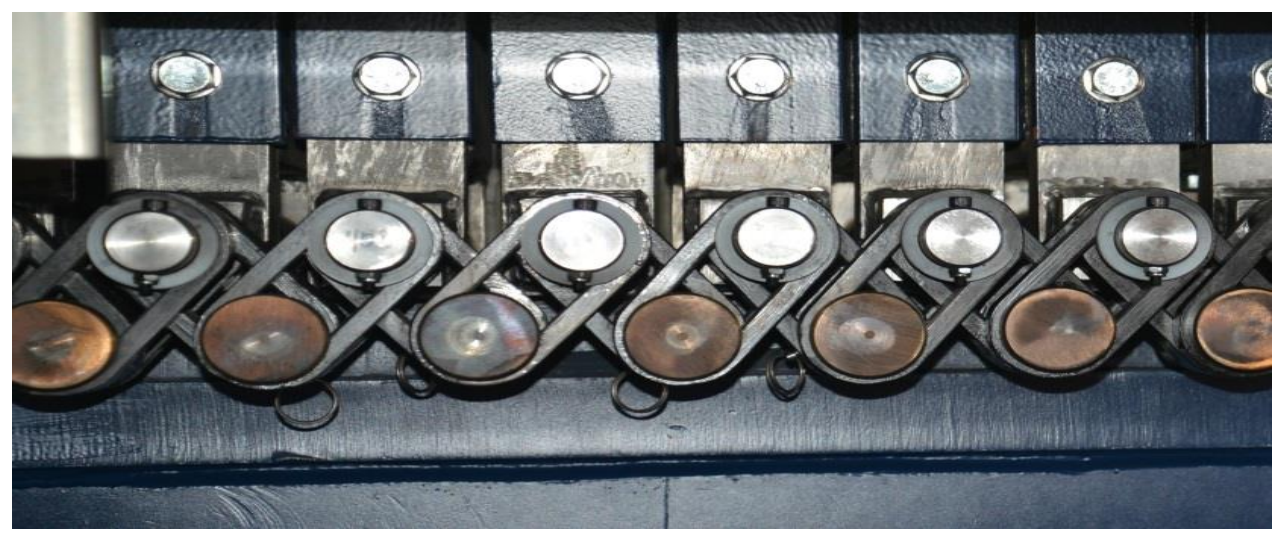

Figure 3 Carbon fibre plate links with filter in closed position

Since commissioning, no links have broken while the filter has been operating under normal conditions. As the filter is not installed in an enclosed and heat controlled building, and the Atacama region of Chile has seen abnormal amounts of snow and freezing conditions this past winter, several links were broken when the filter was started from a shutdown state, during freezing conditions. The cause of these failures was due to the connection pin that connects the links being frozen, not hinging, and the links being crushed as the filter began to close. No failures of this type have been observed during normal operating conditions.

\subsubsection{Large format filter plates}

The size of the filter plates was initially a concern when the Colossal filter was developed. In order for the filter to operate correctly, the slurry would need to fully consolidate in each of the 152 chambers simultaneously to prevent the filter plates from cracking, and to form homogenous, well-formed cakes. For these reasons it was opted to use two industry proven $2,000 \times 2,000 \mathrm{~mm}$ filter plates, welded together to form one monolithic plate.

Since the commencement of commissioning, only eight plates have been damaged by particle erosion. No plates have failed from differential pressures between chambers. The eight plate failures all occurred during the initial start-up of the filter. The damage to the plates was caused by slurry abrasion to the sealing edges of the plates before the cloth washing system was properly calibrated. It is imperative that cloth washing systems are maintained and kept in good working order as experience has shown that regular and effective cloth washing prevents plate damage and extends the media life substantially. Since the washing system has been calibrated, no further damaged plates have been observed.

When designing the filter for the demonstration plant, it was found that to meet the target cake moisture, a filter feed pressure of 15 bar was sufficient, with no air-blow step required. The plates were therefore designed with a four corner discharge configuration, with the upper filtrate ports venting to upper filtrate eyes, but the lower filtrate ports venting to atmosphere from the bottom of the plate. This configuration would allow a supply of compressed air to be fed to the upper filtrate ports, and instead of a traditional air-blow being performed a 'grid sweep', also called 'fast filtering', would be completed instead. Grid sweep is used to remove any remaining filtrate from the grids or 'pips' of the filter plates before the filter is opened to discharge the filter cakes. The process uses the compressed air to push filtrate down the grids and out of the bottom filtrate ports to atmosphere, with no air passing through the filter cake. This step of the cycle is completed in 15 seconds, and uses a very small volume of compressed air, when compared to a traditional air-blow sequence commonly found on filter presses. 
Upon commissioning the filter it was found that the majority of filtrate would flow through the bottom ports of the plate, with very little filtrate flowing upwards to the upper filtrate eyes of the plates. This was a lesson learned, as the plant was designed to take the filtrate from the upper filtrate eyes and route it to an inclined plate clarifier, where it would be cleaned for reuse as filter cloth wash water. With no filtrate flowing from the upper eyes, the wash water system had to be modified to allow for the filtrate that was captured on the drip trays to be diverted to the clarifier. Since the completion of modifications, no further problems have been observed.

\subsubsection{High-pressure feed pumping}

To reduce the cost and cycle time of the filter, it was opted to utilise standard recessed chamber plates, as opposed to membrane filter plates to provide the required 15 bar of driving force to the Colossal filter. By utilising the feed pumps to provide the 15 bar feed pressure, the additional time required to inflate membranes was effectively removed, resulting in a fully consolidated chamber and a reduction in the overall cycle time.

The concern when sizing the pumps was that a medium voltage motor would be required to drive the pump. However, by using two pumps in series, controlled by VFDs, medium voltage motors were not required, and the pumps provide consistent feed pressure which is easy controlled by the filter control system.

When pumping into the filter at high pressures, it should be noted that the seal water system needs to be designed to provide a pressure higher than the internal pump pressure to ensure that flow is maintained at the high operating pressures.

Pump wear has been monitored closely over the past months in an effort to identify any problems that this pumping configuration may present. Since commissioning, two pump sleeves have been replaced due to premature wear. The wear on these sleeves was caused by the freezing of the seal water lines during subzero temperatures. No other issues have been observed with the feed pumping system.

\subsection{Engineering and construction of the demonstration plant}

During the engineering and construction phase of the demonstration plant, several issues were identified. Many of these issues have since been resolved. However, some issues remain and continue to impede the operation of the plant. The following is a list of these issues, including several important lessons that have been learnt.

It is essential that tailings filtration plants are designed with well-planned maintenance areas. The initial demonstration plant maintenance area was designed without services that allowed for the washing of plates during maintenance. Wash water has since been supplied to this area. Since the implementation of these changes, benefits have been seen in the way of cloth life improvement.

During engineering of the plant, historic climate data was reviewed to ensure the design was satisfactory for its intended environment. The plant was then designed as an open structure to save costs. Since commissioning, extensive work has been performed to replace cracked piping and instruments that have been damaged due to freezing temperatures. The operation of the filter has at times been extremely limited due to snowfall (Figure 4), and freezing of the walkways, platforms and filter components. 


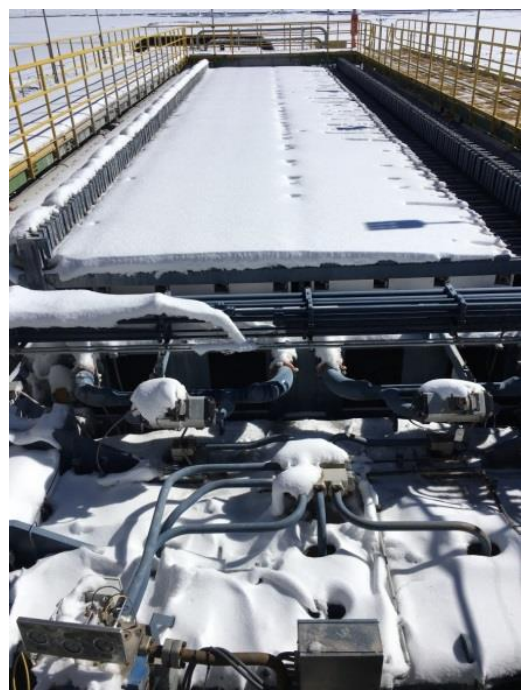

Figure 4 The Colossal filter covered in snow in the Atacama Desert

To reduce capital costs, the plant was designed as an open structure with a semi-mobile gantry crane (Figure 5) to perform maintenance operations. Since commissioning of the plant, maintenance of the filter has been often restricted as the crane cannot be used if the wind speed exceeds $30 \mathrm{~km} / \mathrm{hr}$. This presented itself as a problem during the initial commissioning of the filter, as the filter media that had been damaged by the sun during plant construction required frequent replacement. Since the filter began operating, the damaged media has been replaced, and a routine preventative maintenance schedule has been implemented. It is not uncommon for high wind speeds to be present during the day and because of this, the crane is operated at night while the wind speeds are low. The lesson that can be taken from this demonstration plant is that an enclosed building with a bridge crane is necessary for any full-scale operating plant.

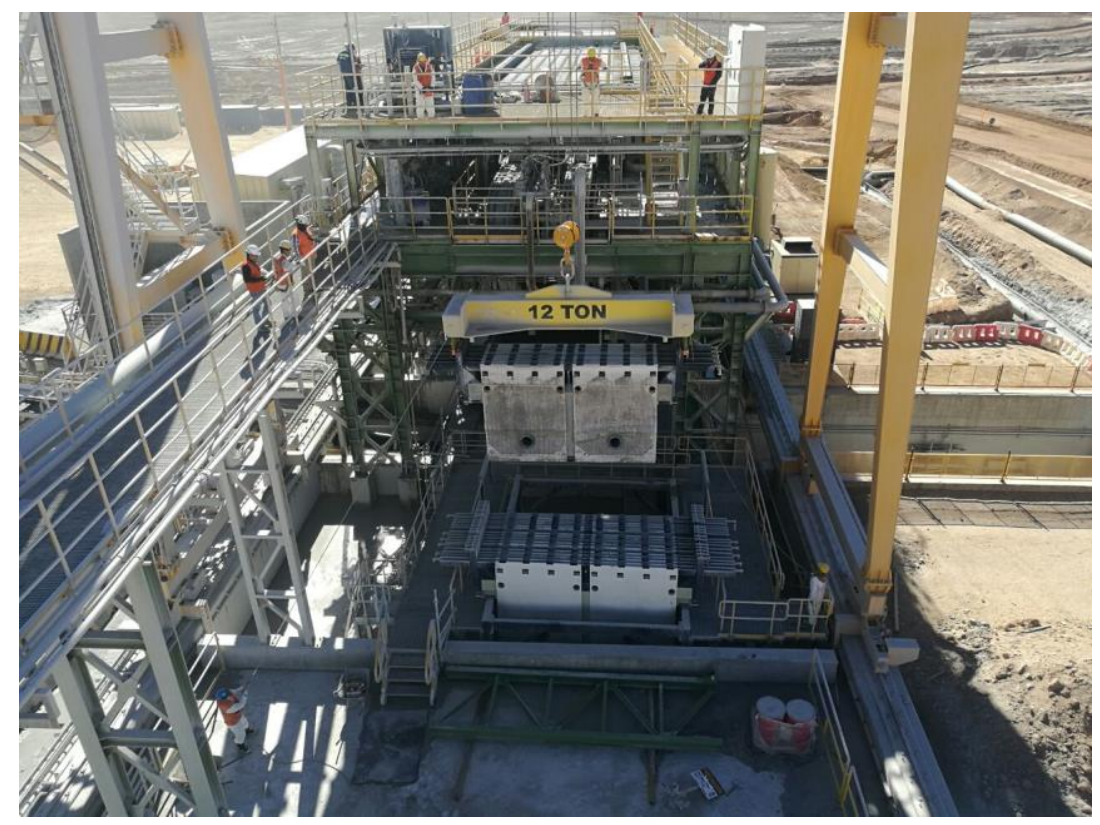

Figure 5 Gantry crane shown lifting filter plates from the maintenance racks to the filter

The plant is fed slurry from the existing tailings thickeners located some distance from the site. After several months of operating the demonstration plant, it was found that the filter feed tank was being fed with extremely inconsistent feed solids. The flow rate of the tailings to the plant also meant that the filter was often sitting dormant for periods of up to four hours, waiting for the filter feed tank to be filled. The problem was found to reside with the tailings pipeline tie-in. In September of 2017, the tie-in was 
upgraded to allow for a higher, more consistent flow of tailings to the demonstration plant. Since this modification, the filter performance and throughput tonnages have increased significantly. For a full-scale plant, a tailings thickener installed at the tailings dewatering facility would overcome these problems.

Throughout the construction phase of this project, the importance of experienced millwrights became apparent. As the size of the filter had increased so significantly, it was extremely important that a solid and true foundation was provided for the installation of the filter. Initially, the foundation provided for the installation of the filter was not within the specified parameters. Experienced millwrights were then used to set the levels, and subsequently the installation and alignment of the filter was completed without any further problems.

\subsection{Filter and HAB feeder operation}

The demonstration plant was first supplied with slurry in June of 2017 - the middle of winter in the Atacama Desert. During initial start-up, freezing weather conditions and snow loading prohibited the operation of the filter and many water pipes, instruments and components were damaged. Once the damaged components were replaced, the filter was restarted and operated.

During the month of October 2017, the production rate of the demonstration plant was ramped up as commissioning continued. During commissioning of the filter, laboratory-scale filtration tests were performed on the feed slurry to determine the optimal filter process parameters. Lab testing was then continued over the period of a month, as the demonstration filter continued to operate. The filter availability from this time forward was able to meet the criteria of $>85 \%$.

When we compare the laboratory filter data from which the plant was designed to the data being provided by the Colossal filter, we see that the Colossal filter is able to produce a slightly drier cake than the laboratory unit, while maintaining the same process times and instantaneous filtration rates. These results provided confirmation that the filter, and all of the design challenges surrounding the high-pressure pumping and filling of the chambers, had been designed correctly. Filtration rates of up to $190 \mathrm{~kg} / \mathrm{hr} / \mathrm{m}^{2}$ were achieved while producing filter cakes between 17 and $19 \mathrm{wt} \%$ cake moisture, with the feed slurry being provided. The feed slurry material at times contained more than $30 \mathrm{wt} \%$ clays, and was typically below $50 \mathrm{wt} \%$ suspended solids. FLSmidth recommends feed solids above $60 \mathrm{wt} \%$ for high filtration rates. Feed slurry with $50 \mathrm{wt} \%$ feed solids contains $50 \%$ more water to process through the filter than a feed slurry containing $60 \mathrm{wt} \%$ feed solids. When being fed with feed slurry with $52 \mathrm{wt} \%$ or greater suspended solids, the Colossal filter was able to achieve its design throughput tonnage. However, low fee solids being supplied to the demonstration plant resulted in longer cycle times, leading to lower daily throughput tonnages.

The HAB feeder, designed to take the impact of the cakes dropping and provide even metering of the material to the takeaway conveyor, has operated without incident since June of 2017. It was initially thought that a metering device would need to be designed for the discharge end of the HAB to provide a more consistent flow of dewatered tailings. However, since commissioning there has been no need to manufacture and implement this device. Dewatered stacks of tailings material can be seen in Figure 6 .

The operation of the filter over the past four months has not been without problems. After two months of operation, premature failure of the bearings used within the cloth shaking mechanism occurred. A design review was completed and new bearings selected. The new bearings have since been installed, and are closely monitored continuously during operation. No further issues have been observed since this modification was completed. 


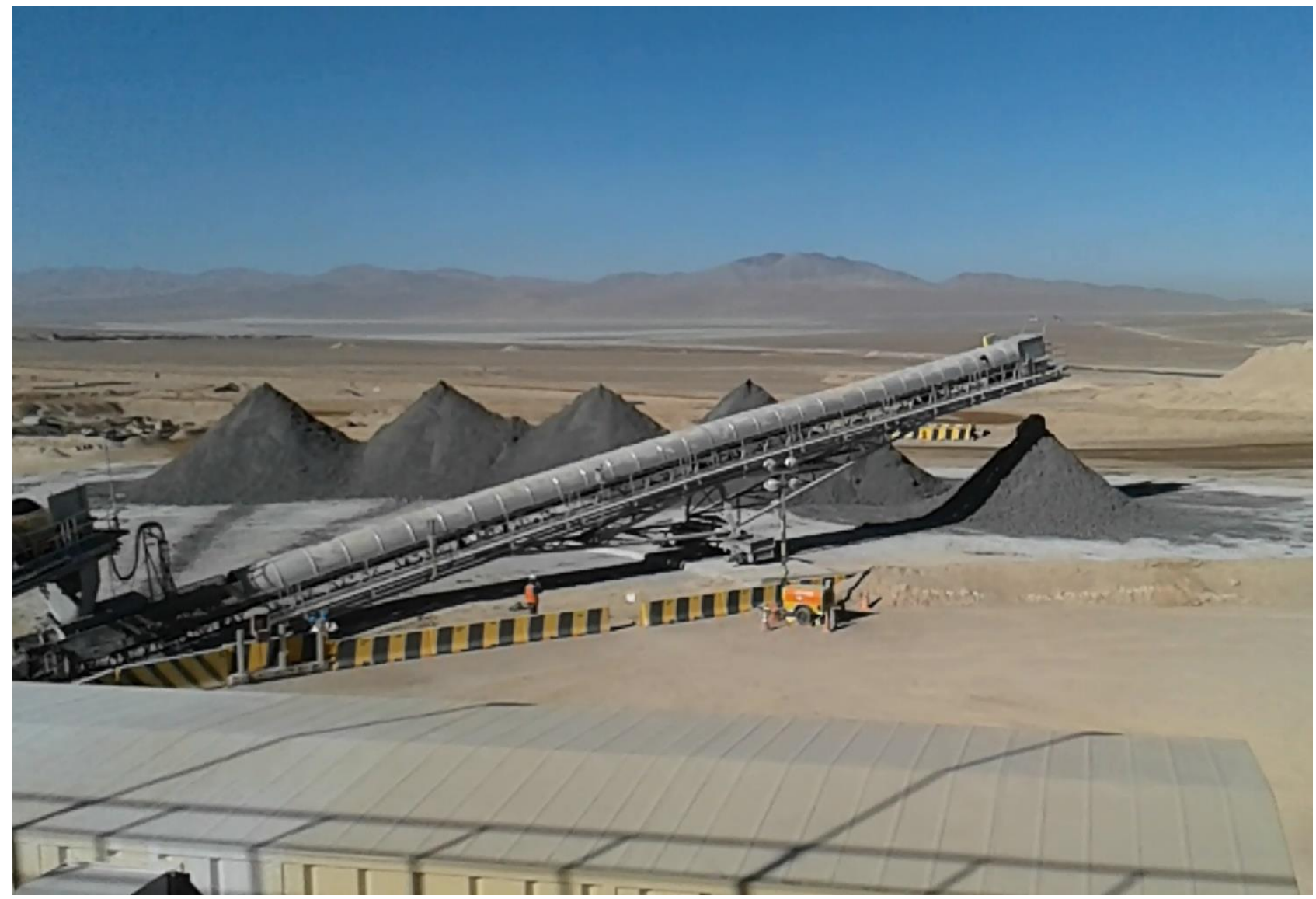

Figure 6 Stacks of dewatered tailings being created by the demonstration plant

\section{Conclusion}

While the development, installation and commissioning of the Colossal filter demonstration plant has not been a trouble-free experience, the challenges that have been faced and overcome have been relatively minor for a development project of such a magnitude. While there are still process improvements to be made with the system, the demonstration plant has already verified that the filter is able to consistently meet the process design parameters. The filter is able to generate cake that consistently meets, or is lower than the target cake moisture, with process times that match the times that were used for sizing of the filter.

While there have been some design issues, the majority of the problems that have been mentioned within this paper do not reside with the filter itself. Instead, the problems reside with the open building design, combined with the abnormal climatic conditions that have been seen this year in the Atacama Desert. The engineering company, performing their due diligence, reviewed historic weather data and wind speed data - these being the driving factors towards making the decision to save capital expenditure, and build an open filtration plant. This is a decision that would not be made again due to the restrictions that it has placed upon the plant when it comes to operation and maintenance of the equipment.

In summary, the Colossal filter demonstration plant has proven that a large format filter can provide $>85 \%$ filter availability while consistently meeting the design target cake moisture and process times. There have been many lessons learned over the duration of this project - lessons which will ensure smoother commissioning and fewer problems on future projects. 
